\title{
Exploring spatial resolution in high-sensitivity nanogap quantum dot photodetectors
}

\author{
Ludan Huang, ${ }^{1}$ Matthew Strathman, ${ }^{2}$ and Lih Y. $\operatorname{Lin}^{2, *}$ \\ ${ }^{1}$ Department of Physics, University of Washington, Seattle, Washington 98195-2500, USA \\ ${ }^{2}$ Department of Electrical Engineering, University of Washington, Seattle, Washington 98195, USA \\ *Corresponding author: lylin@uw.edu \\ Received May 3, 2012; revised June 27, 2012; accepted June 28, 2012; \\ posted June 29, 2012 (Doc. ID 167870); published July 23, 2012
}

\begin{abstract}
We propose a new approach to experimentally determine the spatial resolution of nanogap quantum dot (QD) photodetectors consist of solution-processed QDs. Cross talk between a pair of closely positioned QD photodetectors was measured. Devices with $200 \mathrm{~nm}$ spacing exhibit low crosstalk of 8.4\%. A single QD photodetector also shows high sensitivity, with a lowest detectable optical intensity of $95.3 \mathrm{fW} / \mu \mathrm{m}^{2}$ achieved. The results show the potential of nanogap QD photodetectors for applications in high-density imaging/sensing arrays. ( 2012 Optical Society of America

OCIS codes: $\quad 040.3780,040.5160,160.4236,230.5590,250.3140,350.4238$.
\end{abstract}

As the integration density of photonic integrated circuits progresses toward the submicrometer regime [1], it becomes imperative to detect the optical signal with nanometer resolution as well in order to preserve the on-chip integration density. The requisite photodetection devices are critical for integration of nanophotonics with electronics. The devices should also have high fabrication and integration flexibility with other nanophotonic devices. Furthermore, compatibility with complementary metaloxide-semiconductor (CMOS) manufacturing processes can open other new directions, such as high-resolution imaging and sensing arrays with high sensitivity.

QD photodetectors composed of colloidal QDs have attracted much research attention in recent years due to their many unique advantages, including solution processability, high sensitivity, and high integratability [2,ㅡㄹ. Previously, we have demonstrated nanogap QD photodetectors with high sensitivity, high bandwidth, and high fabrication flexibility [4]. Photoconductivity tunability in nanogap QD photodetectors has also been demonstrated by controlling the trap states [5]. One potential advantage of the nanogap QD photodetectors that has not been extensively studied yet is their prospect to make ultrahigh resolution imaging and sensor arrays. One reason for such potential is that due to solution processing, the device can be easily integrated onto the top of CMOS circuitry, as shown in Fig. 1(a). In contrast to a conventional CMOS image array, where each pixel consists of separate areas for photosensing and control/readout circuit, in a QD photodetector-CMOS chip these two can be vertically integrated. The pixel resolution is ultimately limited by the resolution of CMOS process and the cross talk between the QD photodetectors. In a previous work [6], we have shown by modeling that the $E$-field in such a device structure is highly concentrated in the nanogap region, resulting in a very small active device area, which suggests possible low cross talk even in an ultrahigh density array. In this Letter, we report experimental study of cross talk in closely positioned QD photodetectors.

In typical cross talk characterization, one device is illuminated while the effect on an adjacent device is measured. For example, in a CMOS image array, the effect of cross talk can be investigated by scanning a

micrometer-sized focused light spot over an array of pixels [7] surrounding a central pixel while measuring the response of the central pixel. As the pixel size decreases below the diffraction limit, the conventional approach becomes challenging without resorting to exquisite near-field scanning setups. We propose a new approach to evaluate the cross talk effect in highdensity nanogap QD photodetector arrays. Instead of confining the illumination to an individual photodetector, the entire array is uniformly illuminated with incident light. The photocurrent is monitored in a constantly activated device, while the adjacent device is switched on and off during the measurement. For simplicity, the two devices are referred to as the first device and the second device onward. The cross talk effect is then evaluated by the amount of change in photocurrent induced in the first device when the second device is switched between on and off states. For this study, the cross talk is defined as the amount of change divided by the photocurrent of the first device when the second device is at off state.

To investigate the cross talk effect, we first fabricated pairs of planar nanogap QD photodetectors with various spacing between the photodetectors. The photodetector consists of a thin film of colloidal QDs that fill the gap of a lithographically defined metal nano-junction. The nanogap electrodes, consisting of a $300 \AA$ Au layer with a

(a)

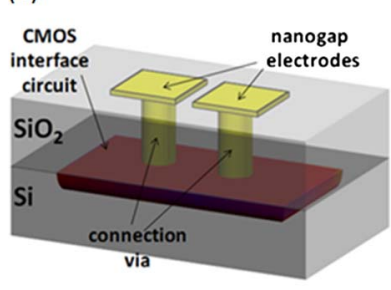

Fig. 1. (Color online) (a) 3D schematics of a nanogap QD photodetector integrated with CMOS circuitry. The QDs cover the surface of the device uniformly and are not shown in the drawing for clarity. (b) SEM image of a pair of nanogap QD photodetectors. The nanogap is $55 \mathrm{~nm}$ and the spacing between the two nanogaps is $\sim 200 \mathrm{~nm}$. The scale bar is $500 \mathrm{~nm}$. The four large electrodes are for probe contacts. 
$20 \AA$ A $\mathrm{Cr}$ adhesion layer, are defined by e-beam lithography (EBL). The electrodes can also be replaced with other metal materials for CMOS process compatibility. The QD film fabrication procedure is similar to that reported in [4]. In brief, CdSe/ZnS core-shell QDs (NNLabs) with $620 \mathrm{~nm}$ peak emission wavelength and octadecyl amine (ODA) ligands are deposited through drop-casting. The devices are then annealed at $300{ }^{\circ} \mathrm{C}$ and $1 \mathrm{mT}$ Torr for $40 \mathrm{~min}$ to improve the charge transport properties between QDs [묘리. Figure $\underline{1(b)}$ shows a scanning electron micrograph (SEM) of a pair of nanogap QD photodetectors. The nanogap is $55 \mathrm{~nm}$, and the separation between the two nanogaps is $200 \mathrm{~nm}$. Drop-casted QDs can be seen covering the surface of the devices with good uniformity. The four large electrodes are for probe contacts during testing. These can be eliminated in integrated devices, shown in Fig. 1(a).

To measure the photocurrent, a Keithley 6430 source meter, also serving as a DC voltage source, is used. Light from a $405 \mathrm{~nm}$ wavelength laser is coupled into an optical fiber and illuminates an array of nanogap QD photodetectors with a spot size of $\sim 100 \mu \mathrm{m}$. The measurement platform is a Cascade M150 low-noise probe station.

From the cross talk measurement result following the procedure described earlier, we discovered that the proposed scheme measures two types of cross talk: optical cross talk and electrical cross talk. Optical cross talk occurs when two active devices are positioned close enough that the active area, determined by the high electricfield area in the nanogap $[4,6]$, of the devices overlaps. Optical cross talk manifests as a decrease in photocurrent in the first device when the second device is switched from off to on, as when active the second device would draw photocurrent from the overlapped active area. Electrical cross talk, on the other hand, arises from modified current paths as a result of biasing the electrodes in the second device. The dark current and the photogenerated carriers in the second device can be detected by the first device. For illustration, we designate the two electrodes in the first device as $E 11$ and $E 12$, and the two electrodes in the second device as $E 21$ and E22, as shown in Fig. 1(b). The applied biases on these four electrodes are noted as $V 11, V 12, V 21$, and $V 22$, respectively. An example of the electrical cross talk effect can be seen by comparing the bias configuration $(V 11, V 12, V 21, V 22)=(5 \mathrm{~V}, 0 \mathrm{~V}, 0 \mathrm{~V}, 0 \mathrm{~V})$ (first device on, second device off) with $(5 \mathrm{~V}, 0 \mathrm{~V}, 5 \mathrm{~V}, 0 \mathrm{~V})$ (both devices on). The source meter connecting $E 11$ and $E 12$ would also measure the current between $E 11$ and $E 21$ in the first configuration due to common ground in the measurement setup. Therefore, the photocurrent in the first configuration is higher than in the second configuration. This is nonetheless indistinguishable from optical cross talk. However, considering the bias configuration $(5 \mathrm{~V}, 0 \mathrm{~V}, 5 \mathrm{~V}, 5 \mathrm{~V})$ (another configuration for first device on, second device off) versus $(5 \mathrm{~V}, 0 \mathrm{~V}, 5 \mathrm{~V}, 0 \mathrm{~V}$ ) (both devices on), an additional electrical path is introduced between $E 11$ and $E 22$ in the second configuration. This results in an increased current for the first device $(E 11)$ when the second device is switched from off to on, an opposite effect of optical cross talk and easily distinguishable. The electrical cross talk effect is present even without the illumination light. Both factors can be utilized to separate electrical cross talk from optical cross talk. In some cases, the measured effect due to electrical cross talk was as high as $25.5 \%$ (average $28 \mathrm{fA}$ change over $110 \mathrm{fA}$ photocurrent) for nanogap QD photodetectors with $\sim 200 \mathrm{~nm}$ spacing.

In the current planar QD photodetector design, the electrodes of the two devices are parallel and elongated in one direction for probing purpose, as shown in Fig. 1(b). This is a significant source of electrical cross talk in the experiment, as there is a large quantity of QDs between the elongated electrodes (high electric-field region during device operation). In future applications of high-density imaging/sensing arrays, the QD photodetectors are expected to be integrated with the underlying CMOS circuitry through holes, as shown in Fig. 1(a). The signal is read out by the embedded circuitry in the CMOS layer, and no extra electrical wiring in the top layer is required. The small electrode areas in the top layer, determined mostly by the nanogap electrodes, will substantially minimize the above-mentioned problem.

In order to obtain a closer estimation for the cross talk in the nanogap QD photodetector arrays integrated with underlying CMOS circuitry without going through multilayer fabrication, we designed a masked planar electrode layout that enables nanoscale electrodes and millimeterscale readout pads on one metal layer by confining the QD deposition areas. Figure 2 depicts the fabrication process. Similar to the plain planar devices, the fabrication starts with $\mathrm{EBL}$ of the electrodes on a $\mathrm{Si} / \mathrm{SiO}_{2}$ wafer, followed by metallization and lift-off, as shown in Figs. 2(a)

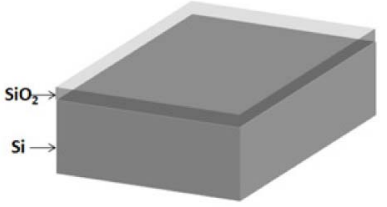

(a)

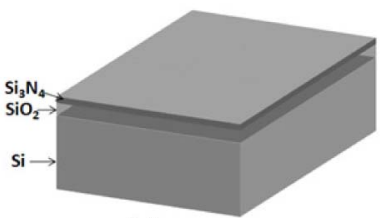

(c)

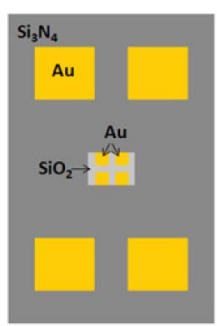

(e)

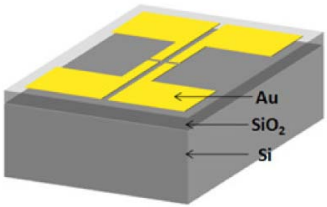

(b)

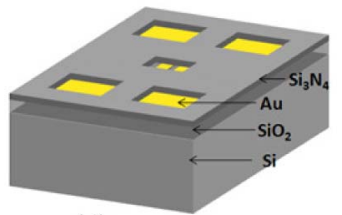

(d)

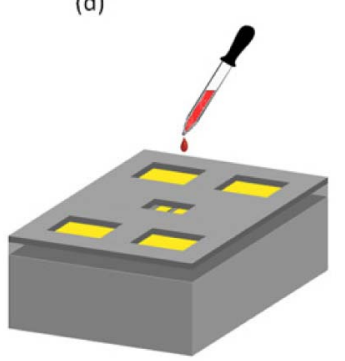

(f)
Fig. 2. (Color online) Fabrication process of $\mathrm{Si}_{3} \mathrm{~N}_{4}$-masked planar nanogap QD photodetectors. A pair of closely spaced devices is shown in the illustration. (a) Starting substrate: $\mathrm{Si}$ with $1 \mu \mathrm{m}$ thick $\mathrm{SiO}_{2}$. (b) Patterning the nanogap electrodes (300 $\AA \mathrm{Au}$ with a $20 \AA \mathrm{Cr}$ adhesion layer) using EBL. (c) Depositing $400 \mathrm{~nm}$ thick $\mathrm{Si}_{3} \mathrm{~N}_{4}$ using PECVD. (d) and (e) Opening windows in the $\mathrm{Si}_{3} \mathrm{~N}_{4}$ layer using EBL and RIE. (f) Drop-casting QDs. 


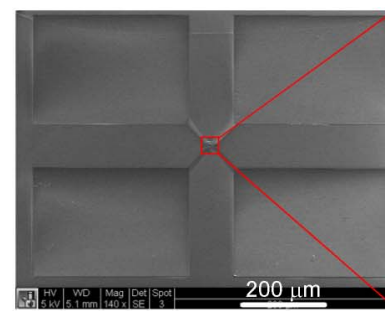

(a)

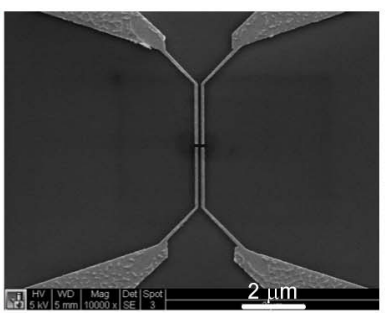

(b)

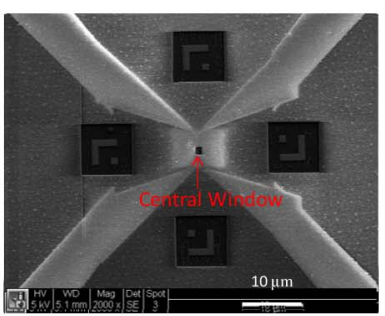

(c)

Fig. 3. (Color online) SEM images of the nanogap electrodes at different steps prior to QD deposition. (a) An overall image of electrodes. (b) A close-up image of the central electrode region [Step Fig. 2(b)]. (c) A close-up image of the central electrode region covered with $\mathrm{Si}_{3} \mathrm{~N}_{4}$ [Step Figs. $\underline{2(\mathrm{~d})}$ and $\underline{2(\mathrm{e})}$ ]. The red arrow points out the central window region.

and 2(b). After this step, instead of depositing QDs directly, a $\mathrm{Si}_{3} \mathrm{~N}_{4}$ layer of $400 \mathrm{~nm}$ thickness is deposited by plasma enhanced chemical vapor deposition (PECVD), as shown in Fig. 2(c). Subsequently, a second EBL is performed to pattern a central window area covering the nanogap electrodes $(800 \mathrm{~nm} \times 1000 \mathrm{~nm})$ and four contact pad areas. After developing the patterns, reactive ion etching (RIE) is performed to etch the exposed $\mathrm{Si}_{3} \mathrm{~N}_{4}$ regions using poly(methyl methacrylate) (PMMA) as the etching mask. Figures $2(\mathrm{~d})$ and $2(\mathrm{e})$ show the resultant three-dimensional and top view of the $\mathrm{Si}_{3} \mathrm{~N}_{4}$-masked planar electrode structure. The central window defines the area for the electrodes, while the four contact pad areas provide access to probe the devices. After this, QDs prepared using the same method described in [4] are drop-cast onto the wafer [Fig. 2(f)]. Through this fabrication procedure, the effective device area is defined by the center window and the electrical cross talk problem can be reduced. Figure 3 shows the SEM images of the electrodes at different steps of fabrication.

We performed cross talk measurements on a pair of $\mathrm{Si}_{3} \mathrm{~N}_{4}$-masked nanogap QD photodetectors spaced $200 \mathrm{~nm}$ apart using the procedure described earlier. As the amount of QDs around the electrodes was much less with the confinement of the $\mathrm{Si}_{3} \mathrm{~N}_{4}$ window, a higher voltage of $20 \mathrm{~V}$ was applied to achieve similar level of photocurrent ( $40 \mathrm{fA})$. An average change of $8.4 \%$ in photocurrent is measured (3.7 fA change over $44 \mathrm{fA}$ ) when the second device is switched from off to on. This is significantly lower than the previous result, showing the effect of confining the QD deposition region in mitigating the electrical cross talk.

We also characterized the sensitivity of the nanogap QD photodetector. Figure 4 shows the photocurrent measurement result versus incident light intensity. The lowest detectable input optical intensity is $95.3 \mathrm{fW} / \mu \mathrm{m}^{2}$, which produces $15.5 \mathrm{fA}$ of photocurrent. Assuming $200 \mathrm{~nm}$ as an acceptable separation distance and each photodetector pixel area is defined as $(200 \mathrm{~nm})^{2}$, this corresponds to $3.8 \mathrm{fW}$ of lowest detectable input power, comparable to a large-area QD photodetector with photoconductive gain [3]. The result shows the utility of the nanogap QD photodetector as a high-sensitivity imaging and sensing device.

In summary, we proposed a scheme to study the cross talk in closely positioned nanogap QD photodetectors. Low cross talk, $\sim 8.4 \%$, is measured for devices spaced

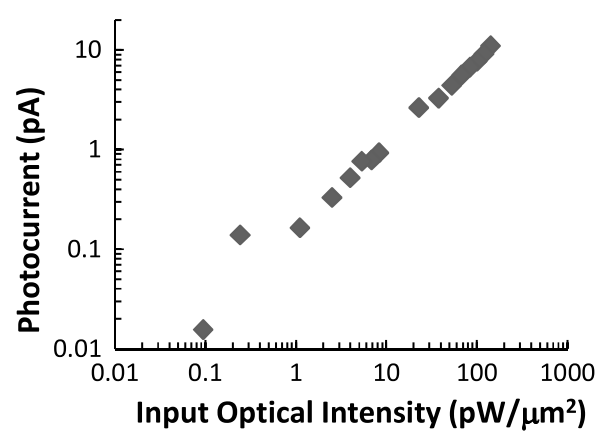

Fig. 4. Photocurrent versus input optical intensity of the nanogap QD photodetector. The lowest detectable intensity is $95.3 \mathrm{fW} / \mu \mathrm{m}^{2}$.

$200 \mathrm{~nm}$ apart. We also measured the sensitivity of a single device. The lowest detectable optical intensity of $95.3 \mathrm{fW} / \mu \mathrm{m}^{2}$ is obtained. The results demonstrate the potential of the nanogap QD photodetectors for high-density imaging/sensing arrays.

This work was supported in part by the National Science Foundation (NSF) (ECCS-0925378 and supplementary GOALI grant), as well as the UW Commercialization Grant Fund. Work was performed in part at the UW Nanotech User Facility (NTUF), a member of the National Nanotechnology Infrastructure Network (NNIN) supported by the National Science Foundation.

\section{References}

1. M. Ohtsu, K. Kobayashi, T. Kawazoe, S. Sangu, and T. Yatsui, IEEE J. Sel. Top. Quantum Electron. 8, 839 (2002).

2. G. Konstantatos, I. Howard, A. Fischer, S. Hoogland, J. Clifford, E. Klem, L. Levina, and E. H. Sargent, Nature 442, 180 (2006).

3. G. Konstantatos, J. Clifford, L. Levina, and E. H. Sargent, Nat. Photon. 1, 531 (2007).

4. M. C. Hegg, M. P. Horning, T. Baehr-Jones, M. Hochberg, and L. Y. Lin, Appl. Phys. Lett. 96, 101118 (2010).

5. L. J. Willis, J. A. Fairfield, T. Dadosh, M. D. Fischbein, and M. Drndic, Nano Lett. 9, 4191 (2009).

6. M. C. Hegg and L. Y. Lin, Opt. Express 15, 17163 (2007).

7. G. Agranov, V. Berezin, and R. H. Tsai, IEEE Trans. Electron Devices 50, 4 (2003).

8. V. J. Porter, S. Geyer, E. Halpert, M. Kastner, and M. Bawendi, J. Phys. Chem. C 112, 2308 (2008).

9. S. Biswas, D. J. Gosztola, G. P. Wiederrecht, M. A. Stroscio, and M. Dutta, J. Electron. Mater. 41, 524 (2012). 